\title{
On Blow-up of Solutions for Quasilinear Degenerate Parabolic Equations
}

\author{
Dedicated to Professor Teruo Ikebe on his sixtieth birthday
}

By

\begin{abstract}
Takashi Ima** and Kiyoshi MochIzUKI**
\end{abstract}
\section{Introduction}

Let $\Omega$ be a bounded domain in $\boldsymbol{R}^{n}$ with smooth boundary $\partial \Omega$. In this paper we consider the parabolic initial-boundary value problem

$$
\begin{array}{lll}
(a(u))_{t}=\Delta u+f(u) & \text { in } & \Omega \times(0, T), \\
B u(x, t)=0 & \text { on } & \partial \Omega \times(0, T), \\
u(x, 0)=u_{0}(x) & \text { in } & \Omega
\end{array}
$$

with the boundary operator

$$
B u(x, t)= \begin{cases}u(x, t) & (\text { Dirichlet) or } \\ \partial_{v} u(x, t) & (\text { Neumann) or } \\ \left\{\partial_{v}+\sigma(x)\right\} u(x, t) & (\text { Robin) } .\end{cases}
$$

Here $v=\left(v_{1}(x), \ldots, v_{\mathrm{n}}(x)\right)$ is the outer unit normal to $\partial \Omega, \partial_{v}=v \cdot \nabla=$ $\sum_{j=1}^{n} v_{j}(x) \frac{\partial}{\partial x_{j}}$ and $\sigma(x)(\neq 0)$ is a nonnegative $C^{1}$-function on $\partial \Omega$.

Throughout this paper we assume the following conditions.

(A1) $a(\xi), \quad f(\xi) \in C^{\infty}\left(\boldsymbol{R}_{+}\right) \cap C\left(\bar{R}_{+}\right) ; \quad a(\xi)>0, \quad a^{\prime}(\xi)>0, \quad f(\xi)>0$ and $f^{\prime}(\xi)>0$ for $\xi>0$, where ' $=d / d \xi$;

(A2) $f_{\circ} a^{-1}$ is locally Lipschitz continuous on $[a(0), \infty)$.

(A3) $u_{0}(x) \in L^{\infty}(\Omega) ; u_{0}(x) \geq 0$ in $\Omega$; the support of $u_{0}(x)$ contains an open nonempty subset of $\Omega$.

These conditions guarantee the unique existence of local (in time) solution $u(x, t) \geq 0$ which satisfies $(0.1) \sim(0.3)$ in a weak sense (cf. e.g., [1],[2],[12] and $[13])$. In the following we do not enter into this existence problem. The definition

Communicated by S. Matsuura. April 11, 1989. Revised December 4, 1990. 1991 Mathematics Subject Classification: 35K65.

* Toyoshina High School, Toyoshina-cho, 399-82, Nagano Prefecture. Japan.

** Department of Mathematics, Faculty of Science, Shinshu University, Matsumoto 390, Japan. 
of "weak" solutions will be given below in $\$ 1$.

Equation (0.1) arises e.g., in the study of thermal diffusion phenomena with heat source. In this case $u(x, t)$ represents a temperature, and there may be several situations which force its maximal value to increase indefinitely in a finite time. The object of this paper is to obtain conditions which ensure the blow-up of solutions. In addition we shall study some behaviours of solutions at the blowup time.

In semilinear cases $a(\xi)=\xi$, these problems have been studied by many authors: see e.g., [3],[5],[6],[8] and [11]. However, there are not so many studies in the above quasilinear cases. We reffer [4],[7],[9] and [10]. Among them in Itaya [9],[10] is treated the initial-boundary value problem for

$$
u_{t}=b(u) u_{x x}+f(u) \text {. }
$$

Assuming $b(\xi)>0$ and $b^{\prime}(\xi) \geq 0$ for all $\xi \geq 0$, he discussed how the coefficient $b(\xi)$ affects the blow-up and nonblow-up properties of solutions.

In $\S 1$ we shall summarize Itaya's results under slightly weaker conditions on coefficients. Let $(s(x), \lambda)$ be the principal eigen-solution of $-\Delta$ in $\Omega$ with boundary condition (0.2) (s is normalized: $\mathrm{s}>0$ and $\int_{\Omega} s(x) d x=1$ ). Put

$$
J(t)=\int_{\Omega} a(u(x, t)) s(x) d x .
$$

Then $J(t)$ goes to infinity in a finite time if the following (A4) is satisfied (Theorem 1.1).

(A4) There exist a function $g(\xi)$ and a $\xi_{0} \geq 0$ such that

$$
\begin{gathered}
g(\xi) \leq f(\xi)-\lambda \xi \text { in } \xi \in \overline{\mathbb{R}}_{+} ; \\
\Gamma=g_{\circ} a^{-1} \text { is convex in }(a(0), \infty) ; \\
g(\xi)>0 \text { and } \int_{\xi}^{\infty} \frac{a^{\prime}(\eta) d \eta}{g(\eta)}<\infty \text { if } \xi>\xi_{0} ; \\
J(0)>a\left(\xi_{0}\right) .
\end{gathered}
$$

Note that these conditions are almost necessary to raise the blow-up. More precisely, the following (A5) or (A6) enables us to obtain apriori estimates (Theorem 1.5) which, with some additional conditions, may imply the global existence of solutions.

(A5) There exist constants $\alpha, \beta \geq 0$ such that

$$
f(\xi) \xi \leq \alpha A(\xi)+\beta \text { in } \xi \geq 0, \text { where } A(\xi)=\int_{0}^{\xi} a^{\prime}(\eta) \eta d \eta .
$$

(A6) (Dirichlet or Robin problem) There exist constants $\beta \geq 0,0 \leq \gamma<\lambda$, 
sufficiently small $\delta \geq 0$ and $1 \leq q \leq \frac{n+2}{n-2}(1 \leq q<\infty$ if $n=1,2)$ such that

$$
F(\xi) \equiv \int_{0}^{\xi} f(\eta) d \eta \leq \beta+\frac{\gamma \xi^{2}}{2}+\frac{\delta \xi^{q}}{q+1} \text { in } \xi \geq 0 .
$$

In $\S 2$ we shall employ methods of Friedman-McLeod [5] (cf. also ChenMatano [3] and Fujita-Chen [6]) to obtain several properties of solutions near the blow-up time, say $t=T$.

An interesting result is obtained for the Neumann problem. Let $n=1$ and assume that

$$
F(\xi)=\mathrm{o}\left(\xi^{2}\right) \quad \text { as } \xi \rightarrow \infty .
$$

Then every solution $u(x, t)$ blows up on the whole interval $\bar{\Omega} \times\{T\}=\{(x, T)$; $x \in[0, L]\}$, and we have

$$
\lim _{t \uparrow T} \frac{u(x, t)}{\Theta^{-1}(T-t)}=1 \quad \text { for any } x \in \bar{\Omega},
$$

where

$$
\Theta(\xi)=\int_{\xi}^{\infty} \frac{a^{\prime}(\eta) d \eta}{f(\eta)}
$$

(Theorem 2.2). This result is typical to our Neumann problem. Note that $(0.11)$ and (A4) contradicts each other provided the Dirichlet or Robin problem is concerned with. In case of semilinear equations, the contradiction occurs even for the Neumann problem.

In contrast with $(0.11)$ if we assume

$$
a^{\prime \prime}(\xi) \leq 0, f^{\prime \prime}(\xi)>0 \text { and } \int_{\xi}^{\infty} f(\eta)^{-\delta} d \eta<\infty, \text { where } 0<\delta<1,
$$

then the blow-up situation is completely changed. In this case, with some additional conditions, the so-called single point blow-up occurs (Corollary 2.5) as in the case of semilinear equations.

Finally, we note that the Cauchy problem for $(0.1)$ is studied in the recent work of Suzuki [14], where asymptotic behaviours of the free boundary is discussed near the blow-up time.

\section{§1. Blow-up and Nonblow-up Properties}

By a solution of the initial-boundary value problem $(0.1) \sim(0.3)$ we mean a function $u(x, t)$ in $\bar{\Omega} \times[0, T)$ such that

(i) $u(x, t) \in C\left([0, T) ; L^{\infty}(\bar{\Omega})\right)$, 
(ii) $u(x, t) \geq 0$ for each $(x, t) \in \bar{\Omega} \times[0, T)$,

(iii) $u(x, t)$ satisfies

$$
\left.\int_{\Omega} a(u(x, t)) \varphi(x, t) d x\right|_{\left.\right|_{t}=t_{0}} ^{t=t_{1}}=\int_{t_{0}}^{t_{1}} \int_{\Omega}\left\{a(u) \varphi_{t}+u \Delta \varphi+f(u) \varphi\right\} d x d t
$$

for any $0 \leq t_{0}<t_{1}<T$ and nonnegative $\varphi \in C^{2}(\Omega \times[0, T))$ verifying the boundary condition $B \varphi(x, t)=0$ on $\partial \Omega \times[0, T)$.

This "weak" solution is the limit of a monotone nonincreasing sequence of classical solutions for regularized problems of $(0.1) \sim(0.3)$. So the usual comparison theorem holds. Moreover, if $u_{0}(x)>0$ in $\Omega$, the corresponding solution is classical.

For each solution $u(x, t)$ we define $J(t)$ by $(0.4)$. Then by (i) $J(t)$ is continuous in $t>0$, and by (iii)

$$
J(t)-J(0)=\int_{0}^{t} \int_{\Omega}\{-\lambda u(x, \tau)+f(u(x, \tau)\} s(x) d x d \tau
$$

since $s \in C^{2}(\Omega)$ and satisfies the boundary condition.

Theorem 1.1. We assume (A4). Then there exists a $T>0$ such that

$$
J(t) \rightarrow \infty \text { as } t \uparrow T \text {. }
$$

Proof. Let $g$ be as given in (A4). Then from (1.1)

$$
J(t) \geq J(0)+\int_{0}^{t} \int_{\Omega} g(u(x, \tau)) s(x) d x d \tau .
$$

Moreover, by $(0.6)$, we can use the Jensen inequality to obtain

$$
J(t) \geq J(0)+\int_{0}^{t} \Gamma(J(\tau)) d \tau \equiv \alpha(t) .
$$

Note here $\Gamma(\rho)>0$ in $\rho>a\left(\xi_{0}\right)$. Then (1.3) and the continuity of $J(t)$ show that $\alpha(t) \geq J(0)$ and is increasing in $t>0$. Since $\Gamma(\rho)$ is also increasing in $\rho>a\left(\xi_{0}\right)$, we obtain from $(1.3)$ and $(0.8)$

$$
\Gamma(J(t)) \geq \Gamma(\alpha(t))
$$

or equivalently

$$
1 \leq \Gamma(J(t)) / \Gamma(\alpha(t)) .
$$

Integrate the both sides over $(0, t)$. Noting $d \alpha(t)=\Gamma(J(t)) d t$ and $(0.7)$, we have

$$
t \leq \int_{J(0)}^{\alpha(t)} \frac{d \rho}{\Gamma(\rho)} \leq \int_{J(0)}^{\infty} \frac{d \rho}{\Gamma(\rho)}=\int_{\xi_{1}}^{\infty} \frac{a^{\prime}(\eta) d \eta}{g(\eta)}<\infty
$$

where $\xi_{1}=a^{-1}(J(0))>\xi_{0}$. These inequalities lead to a contradiction unless 


$$
\alpha(T)=J(0)+\int_{0}^{T} \Gamma(J(\tau)) d \tau=\infty \text { for some } T>0
$$

Thus, (1.2) in concluded.

Example 1.2. Let

$$
a(\xi)=\xi^{1 / m} \text { and } f(\xi)=\mu \xi^{p / m}, \text { where } p, m, \mu>0 .
$$

(1) (Dirichlet or Robin problem) Assume $p>\max \{1, m\}$. Then (A4) holds if we choose

$$
g(\xi)= \begin{cases}f(\xi)-\lambda \xi, & \xi \geq\left(\frac{\lambda m}{\mu p}\right)^{m /(p-m)} \\ -\frac{\mu(p-m)}{m}\left(\frac{\lambda m}{\mu p}\right)^{p /(p-m)}, & 0 \leq \xi<\left(\frac{\lambda m}{\mu p}\right)^{m /(p-m)},\end{cases}
$$

$\xi_{0}=(\lambda / \mu)^{m /(p-m)}>0$ (the positive solution of $\left.f(\xi)-\lambda \xi=0\right)$ and $J(0)>a\left(\xi_{0}\right) \equiv$ $(\lambda / \mu)^{1 /(p-m)}$.

(2) (Dirichlet or Robin problem) Assume $p=m>1$ and $\mu>\lambda$. Then (0.8) is automatic, and (A4) holds if we choose

$$
g(\xi)=f(\xi)-\lambda \xi=(\mu-\lambda) \xi
$$

and $\xi_{0}=0$ (cf., Example 1.7 (5) stated below).

(3) (Neumann problem) Assume $p>1$. Then (A4) holds if we choose $g(\xi)=f(\xi)$ and $\xi_{0}=0((0.8)$ is also automatic $)$.

Next we assume (A5) or (A6), and obtain apriori estimates which will play an important role in the study of nonblow-up properties of solutions.

Proposition 1.3. Let $u(x, t)$ be a classical solution of $(0.1) \sim(0.3)$. Then we have for any $t_{0}, \iota_{1} \in(0, T)$,

$$
\begin{aligned}
\text { (1.5) }\left.\int_{\Omega} A(u(x, t)) d x\right|_{t_{0}} ^{t_{1}} & =\int_{t_{0}}^{t_{1}}\left[-\int_{\partial \Omega} \sigma u^{2} d S d t+\int_{\Omega}\left\{-|\nabla u|^{2}+f(u) u\right\} d x\right] d t, \\
\text { (1.6) } 2 \int_{t_{0}}^{t_{1}} \int_{\Omega} a^{\prime}(u) u_{t}^{2} d x d t= & {\left[-\int_{\partial \Omega} \sigma(x) u(x, t)^{2} d S\right.} \\
& +\int_{\Omega}\left\{-|\nabla u(x, t)|^{2}+2 F(u(x, t)\} d x\right]_{t=t_{0}}^{t=t_{1}},
\end{aligned}
$$

where $A$ and $F$ are as given in (0.9) and (0.10), respectively. The surface integrals in the right hand sides vanish if the Dirichlet or Neumann problem is concerned with. 
Proof. (1.5) is obtained if we multiply both sides of $(0.1)$ by $u(x, t)$ and integrate by parts over $\Omega \times\left(t_{0}, t_{1}\right)$. (1.6) is similarly obtained if we multiply $(0.1)$ by $2 u_{t}(x, t)$.

Lemma 1.4. Let $u(x, t)$ be as in Proposition 1.3. We fix any $0<\tau<T$. Then for any $t \in(\tau, T)$,

$$
\begin{gathered}
\int_{\Omega}|\nabla u(t)|^{2} d x+\int_{\partial \Omega} \sigma u(t)^{2} d S \leq C_{\tau}+2 \int_{\Omega} F(u(t)) d x \\
C_{\tau}=\tau^{-1}\left[\int_{\Omega} A\left(u_{0}(x)\right) d x+\int_{0}^{\tau} \int_{\Omega} f(u) u d x d t\right] .
\end{gathered}
$$

Proof. The left side of (1.7) being continuous in $t \in(0, T)$, we can choose $\tau_{0} \in(0, \tau)$ to satisfy

$$
\int_{\Omega}\left|\nabla u\left(\tau_{0}\right)\right|^{2} d x+\int_{\partial \Omega} \sigma u\left(\tau_{0}\right)^{2} d S \leq \tau^{-1} \int_{0}^{\tau}\left[\int_{\Omega}|\nabla u(t)|^{2} d x+\int_{\partial \Omega} \sigma u(t)^{2} d S\right] d t \leq C_{\tau} .
$$

Here for the last inequality we have used (1.5) with $t_{0}=0$ and $t_{1}=\tau$. Substituting the result into (1.6) with $t_{0}=\tau_{0}$ and $t_{1}=t$, we obtain (1.7).

Theorem 1.5. Suppose that the solution $u(x, t)$ of $(0.1) \sim(0.3)$ exists in the interval $(0, T)$. We fix any $0<\tau<T$.

(1) If we assume (A5), then there exists a $C_{1}(\tau, T)>0$ such that

$$
\int_{\Omega}\left\{A(u(t))+|\nabla u(t)|^{2}\right\} d x+\int_{\partial \Omega} \sigma u(t)^{2} d S \leq C_{1}(\tau, T)
$$

for any $t \in(\tau, T)$.

(2) If we assume (A6), then there exists a $C_{2}(\tau)>0$ such that

$$
\int_{\Omega}\left\{u(t)^{2}+|\nabla u(t)|^{2}\right\} d x+\int_{\partial \Omega} \sigma u(t)^{2} d S \leq C_{2}(\tau)
$$

for any $t \in(\tau, T)$.

Proof. We shall prove the assertions for classical solutions. In general cases we can apply a limit procedure.

(1) It follows from (0.9) and (1.5) that

$$
\int_{\Omega} A(u(x, t)) d x \leq C_{3}+\int_{0}^{t} \int_{\Omega}\{\alpha A(u(x, \xi))+\beta\} d x d \tau,
$$

where $C_{3}=\int_{\Omega} A\left(u_{0}(x)\right) d x$. Thus, by use of the Gronwall inequality 


$$
\int_{\Omega} A(u(x, t)) d x \leq\left(C_{3}+\beta|\Omega| / \alpha\right) e^{\alpha t},
$$

where $|\Omega|=\int_{\Omega} d x$. Next, since we have $F(\xi) \leq f(\xi) \xi$, it follows from (0.9) and (1.7) that

$$
\int_{\Omega}|\nabla u(t)|^{2} d x+\int_{\partial \Omega} \sigma u(t)^{2} d S \leq C_{\tau}+\int_{\Omega}\{\alpha A(u(t))+\beta\} d x
$$

for any $t \in(\tau, T)$. (1.11) and (1.12) prove (1.9).

(2) Put

$$
M(t) \equiv \int_{\Omega}|\nabla u(x, t)|^{2} d x+\int_{\partial \Omega} \sigma(x) u(x, t)^{2} d S .
$$

Then since $\lambda>0$ is the principal eigenvalue of $-\Delta$ with boundary condition on $\partial \Omega$, we have

$$
\int_{\Omega} u(x, t)^{2} d x \leq \lambda^{-1} M(t) .
$$

Hence, it is enough to show the boundedness of $M(t)$ in $t \in(\tau, T)$. By means of $(0.10)$

$$
\int_{\Omega} F(u(t)) d x \leq \beta|\Omega|+\frac{\gamma}{2} \int_{\Omega} u(t)^{2} d x+\frac{\delta}{q+1} \int_{\Omega} u(t)^{q+1} d x .
$$

Thus, by (1.14) and the Sobolev embedding

$$
\int_{\Omega} F(u(t)) d x \leq \beta|\Omega|+\frac{\gamma}{2 \lambda} M(t)+\frac{\delta}{q+1} C M(t)^{(q+1) / 2} .
$$

This and (1.7) imply

$$
\widetilde{C}_{\tau}-\frac{\lambda-\gamma}{\lambda} M(t)+\frac{2 \delta}{q+1} C M(t)^{(q+1) / 2} \geq 0,
$$

where $\widetilde{C}_{\tau}=C_{\tau}+2 \beta|\Omega|$. Consider the function

$$
N=\widetilde{C}_{\tau}-\frac{\lambda-\gamma}{\lambda} M+\frac{2 \delta}{q+1} C M^{(q+1) / 2}
$$

of $M \geq 0$. Since $\delta$ is assumed very small, $N$ becomes negative for certain values of $M>0$. Let $M_{\tau}>0$ be the smallest value of $M$ where $N$ vanishes. Since $M(0) \leq \widetilde{C}_{\tau}<\frac{\lambda-\gamma}{\lambda} M_{\tau}<M_{\tau}$, by continuity of $M(t)$ in $t, M(t)$ is not larger than $M_{\tau}$. 
Corollary 1 . (0.1) (0.3) exists globally in time.

Proof. In case of the Dirichlet or Robin problem, the Sobolev embedding shows that

$$
\sup _{\mathrm{x} \in \Omega} u(x, t) \leq \widetilde{C}\|\nabla u(\cdot, t)\|_{L^{2}}
$$

This and Theorem $1.5(1)$ or (2) guarantee that $u(x, t)$ is apriori bounded in $L^{\infty}(\Omega)$. Thus, every local solution can be prolonged up to any time $T>0$.

In case of the Neumann problem, we have by Theorem 1.5 (1)

$$
u(x, t)-|\Omega|^{-1} \int_{\Omega} u(x, t) d x \leq \widetilde{C} C_{1}(\tau, T)^{1 / 2}
$$

Moreover, since $\int_{\Omega} A(u(x, t)) d x \leq \mathbb{C}_{1}(\tau, T)$, the mean value of solution, and hence, $u(x, t)$ itself are necessarily bounded in $\tau<t<T$. Thus, every local solution is also prolonged up to any time $T>0$.

Example 1.7. Let $a(\xi), f(\xi)$ be as given in (1.4).

(4) Assume $p \leq 1$. Then (A5) holds if we choose $\alpha=\mu(m+p)$ and $\beta=\frac{\mu(1-p)}{m+1}$.

(5) Assume $\mu<\lambda$ and $1<p=m$ [or $\mu>0$ and $1<p<m]$. Then (A6) holds if we choose $\gamma=\mu$ and $\beta=\delta=0$ [or any $0<\gamma<\lambda, \beta=\frac{\gamma(m-p)}{2(m+p)}\left(\frac{\mu}{\gamma}\right)^{2 m /(m-p)}$ and $\delta=0]$.

(6) Assume $1<\frac{p}{m} \leq \frac{n+2}{n-2}\left(1<\frac{p}{m}<\infty\right.$ if $\left.n=1,2\right)$ and $\mu$ being sufficiently small. Then (A6) holds if we choose $\beta=\gamma=0, \delta=\mu$ and $q=p / m$.

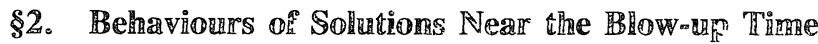

In this section we assume (A4) and consider solutions of $(0.1) \sim(0.3)$ near the blow-up time $t=T$. Here $T$ is defined by

$$
T=\sup \{\tau ; u(x, t) \text { exists for all }(x, t) \in \Omega \times(0, \tau)\} .
$$

This implies $\lim _{t \uparrow T} \sup _{x \in \Omega} u(x, t)=\infty$. In this case by a blow-up point of $u(x, t)$ we mean a point $x \in \bar{\Omega}$ such that there exists a sequence $\left(x_{m}, t_{m}\right) \in \Omega \times(0, T)$ satisfying

$$
x_{m} \rightarrow x, t_{m} \uparrow T \text { and } u\left(x_{m}, t_{m}\right) \rightarrow \infty \text { as } m \rightarrow \infty \text {. }
$$

We put 


$$
U(t)=\sup _{x \in \Omega} u(x, t), \quad V(t)=\inf _{x \in \Omega} u(x, t)
$$

and

$$
\Theta(\xi)=\int_{\xi}^{\infty} \frac{a^{\prime}(\eta) d \eta}{f(\eta)} \text { for } \xi>0 .
$$

Obviously $\Theta(\xi)>0$ and decreases to 0 as $\xi \rightarrow \infty$.

Theorem 2.1. (1) We have

$$
U(t) \geq \Theta^{-1}(T-t) \quad \text { near } t=T .
$$

(2) Suppose that $V(t) \rightarrow \infty$ as $t \uparrow T$. Then we have

$$
V(t) \leq \Theta^{-1}(T-t) \quad \text { near } t=T \text {. }
$$

Proof. Note that $u(x, t)$ satisfies $(0.1)$ in the classical sense at each point $(x, t) \in \Omega \times(0, T)$ where $u(x, t)>0$. Thus, to show this theorem we can assume $u(x, t)$ is a classical solution.

(1) Let $U\left(t_{i}\right)=u\left(x_{i}, t_{i}\right), i=1,2$. Then since

$$
\begin{aligned}
& a\left(U\left(t_{2}\right)\right)-a\left(U\left(t_{1}\right)\right) \leq a\left(u\left(x_{2}, t_{2}\right)\right)-a\left(u\left(x_{2}, t_{1}\right)\right), \\
& a\left(U\left(t_{2}\right)\right)-a\left(U\left(t_{1}\right)\right) \geq a\left(u\left(x_{1}, t_{2}\right)\right)-a\left(u\left(x_{1}, t_{1}\right)\right),
\end{aligned}
$$

it follows that $a(U(t))$ is Lipschitz continuous and

$$
\frac{a\left(U\left(t_{2}\right)\right)-a\left(U\left(t_{1}\right)\right)}{t_{2}-t_{1}} \leq a\left(u\left(x_{2}, t_{2}\right)\right)_{t}+o(1)=\Delta u\left(x_{2}, t_{2}\right)+f\left(u\left(x_{2}, t_{2}\right)\right)+o(1)
$$

if $t_{2}>t_{1}$. Since $\Delta u\left(x_{2}, t_{2}\right) \leq 0$, letting $t_{1} \rightarrow t_{2}$, we obtain

$$
a(U(t))^{\prime} \leq f(U(t)) \text { for a.e. } t \in(0, T),
$$

or equivalently

$$
1 \geq a(U(t))^{\prime} / f(U(t)) \text { for a.e. } t \in(0, T),
$$

where ' $=d / d t$. Integrate the both sides over $(t, T)$ and note that $U(\tau) \rightarrow \infty$ as $\tau \uparrow T$. Then

$$
T-t \geq \int_{t}^{T} \frac{a(U(\tau))^{\prime} d \tau}{f(U(\tau))}=\int_{U(t)}^{\infty} \frac{a^{\prime}(\eta) d \eta}{f(\eta)}=\Theta(U(t)) .
$$

$\Theta$ being monotone decreasing, this shows (2.3).

(2) Let $V\left(t_{i}\right)=u\left(x_{i}, t_{i}\right), i=1,2$. Then we have similarly

$$
\frac{a\left(V\left(t_{2}\right)\right)-a\left(V\left(t_{1}\right)\right)}{t_{2}-t_{1}} \geq a\left(u\left(x_{2}, t_{2}\right)\right)^{\prime}+o(1)=\Delta u\left(x_{2}, t_{2}\right)+f\left(u\left(x_{2}, t_{2}\right)\right)+o(1)
$$


if $t_{2}>t_{1}$. By assumption $V(\tau) \rightarrow \infty$ as $\tau \uparrow T$. Thus, starting from this inequality, we can follow the above proof to get $(2.4)$.

Theorem 2.2. We consider the Neumann problem. Let $n=1$ and assume in addition to (A4) that

(A7) $F(\xi) \leq\left\{(\varepsilon \xi)^{2}+\beta\right\} / 2 L^{2}$ for some $0<\varepsilon<1$ and $\beta \geq 0$. Then $u(x, t)$ blows up at any point in $\bar{\Omega}=[0, L]$. Moreover, for any $\varepsilon<\varepsilon^{\prime}<1$ there exists a $0<\tau<T$ such that

$$
1-\varepsilon^{\prime} \leq \frac{u(x, t)}{\Theta^{-1}(T-t)} \leq \frac{1}{1-\varepsilon^{\prime}} \text { for any }(x, t) \in[0, L] \times(\tau, T) .
$$

Remark. For the Dirichlet or Robin problem, (A7) with $\varepsilon^{2}<\lambda L^{2}$ gives a nonblow-up condition.

Proof. Let $V(t)=u\left(x_{t}, t\right)$. Then by (1.7)

$$
\begin{aligned}
u(x, t)-V(t) & =\int_{x_{t}}^{x} u_{x}(y, t) d x \leq\left(L^{-1} \int_{0}^{\mathrm{L}}\left|u_{x}(x, t)\right|^{2} d x\right)^{1 / 2} \\
& \leq\left[L\left\{C_{\tau}+2 \int_{0}^{L} F(U(t)) d x\right\}\right]^{1 / 2}
\end{aligned}
$$

Applying (A7) to the right, we have

$$
u(x, t)-V(t) \leq \varepsilon U(t)+C_{\tau, \beta},
$$

where $C_{\tau, \beta}=\left(L C_{\tau}+\beta\right)^{1 / 2}$. This implies

$$
(1-\varepsilon) U(t) \leq V(t)+C_{\tau, \beta}
$$

From Theorem 2.1 (1) it follows that $V(t) \rightarrow \infty$ as $t \uparrow T$, and hence $u(x, t)$ blows up at any point $x \in[0, L]$. Moreover, since

$$
(1-\varepsilon) U(t)-C_{\tau, \beta} \leq u(x, t) \leq \frac{1}{1-\varepsilon}\left\{V(t)+C_{\tau, \beta}\right\},
$$

by use of Theorem 2.1 (1) and (2), we conclude (2.5).

The above theorem asserts that $u(x, t)$ blows up uniformly in $\Omega$ :

$$
\lim _{t \uparrow T x \in \Omega} \inf u(x, t)=\infty \text {. }
$$

It may be interesting to compare this with results on the so-called single point blow-up.

In the following we restrict ourselves to the case $n=1$ and $\Omega=(0, L)$, and follow the argument of [3],[5],[6] developed for semi-linear blow-up problems. We require (A4) and 
(A8) There exists a $\xi_{1}>0$ such that $a^{\prime \prime}(\xi) \leq 0$ in $\xi \geq \xi_{1}$.

Lemma 2.3. Let $0<\tau<T$ and $\omega_{0}=\left(x_{0}, y_{0}\right) \subset(0, L)$. Suppose that

$$
u_{x}(x, t)<0[\text { or }>0] \text { in } \omega_{0} \times[\tau, T) .
$$

If $u(x, t)$ blows up at a point $z \in \omega_{0}$, then it blows up uniformly in each interval $\omega=(\bar{x}, \bar{y}) \subset \subset\left(x_{0}, z\right)\left[\operatorname{or} \subset \subset\left(z, y_{0}\right)\right]$.

Proof. We shall consider only the case

$$
u_{x}(x, t)<0 \text { in } \omega_{0} \times[\tau, T) .
$$

By definition there exist sequences $\left\{z_{m}\right\}$ and $\left\{t_{m}\right\}$ such that $z_{m} \rightarrow z, t_{m} \uparrow T$ and $a_{m}=u\left(z_{m}, t_{m}\right) \rightarrow \infty$ as $m \rightarrow \infty$. We fix any $\bar{y}<x_{1}<z$. Without loss of generality, we can assume $\left\{z_{m}\right\} \in\left(x_{1}, y_{0}\right)$. We put

$$
v(x, t)=\eta_{m}(t) \sin \left\{\frac{\pi}{\delta}\left(x-x_{0}\right)\right\} \quad \text { in } Q_{m} \equiv\left(x_{0}, x_{1}\right) \times\left(t_{m}, \infty\right),
$$

where $\delta=x_{1}-x_{0}$ and

$$
\eta_{m}(t)=W^{-1}\left\{W\left(a_{m}\right)-\left(\frac{\pi}{\delta}\right)^{2}\left(t-t_{m}\right)\right\} \quad \text { with } W(s)=\int_{1}^{s} \frac{a^{\prime}(\xi)}{\xi} d \xi
$$

$v(x, t)$ is a subsolution to $(0.1)$ in $Q_{m}$ since $\eta_{m}(t)>0$ satisfies

$$
\eta^{\prime}=-\left(\frac{\pi}{\delta}\right)^{2} \frac{\eta}{a^{\prime}(\eta)} \text { in } t>t_{m} \text { with } \eta\left(t_{m}\right)=a_{m}
$$

and $a^{\prime}\left(\eta_{m}(t)\right) \leq a^{\prime}(v(x, t))$ by (A8) and (2.8). Noting $(2.7)^{\prime}$, we have

$$
\begin{array}{ll}
u\left(x, t_{m}\right) \geq a_{m} \geq v\left(x, t_{m}\right) & \text { in } x \in\left(x_{0}, x_{1}\right), \\
u\left(x_{i}, t\right) \geq 0=v\left(x_{i}, t\right) & \text { in } t \in\left(t_{m}, T\right)(i=0,1) .
\end{array}
$$

Thus, by a comparison theorem

$$
u(x, t) \geq \eta_{m}(t) \sin \left\{\frac{\pi}{\delta}\left(x-x_{0}\right)\right\} \text { in }\left(x_{0}, x_{1}\right) \times\left(t_{m}, T\right) .
$$

As is easily seen, $\eta_{m}(t) \geq \eta_{m}(T)$ in $t_{m} \leq t<T$ and it follows from (2.9) that $\eta_{m}(T) \rightarrow \infty$ as $m \rightarrow \infty$. This and (2.10) show the assertion.

Let $u(x, t)$ be a classical solution of $(0.1)$ in a subdomain $\omega_{0} \times(\tau, T)$ of $\Omega \times$ $(0, T)$. For sufficiently smooth $\rho(x), x \in \omega_{0}$, and $\Phi(\xi), \xi>0$, we put

$$
J(x, t)=u_{x}(x, t)+\rho(x) \Phi(u(x, t)) .
$$

By a direct calculation we see that this $J$ satisfies the equation 


$$
\left[a^{\prime}(u) J\right]_{t}-J_{x x}=A(x, t) J_{x}+B(x, t) J+C(x, t)+D(x, t)
$$

in $\omega_{0} \times(\tau, T)$, where

$$
\begin{aligned}
A(x, t)= & \frac{\rho(x) a^{\prime \prime}(u) \Phi(u)}{a^{\prime}(u)}, \\
B(x, t)= & f^{\prime}(u)-2 \rho^{\prime}(x) \Phi^{\prime}(u)-\frac{\rho(x)^{2} a^{\prime \prime}(u) \Phi(u) \Phi^{\prime}(u)}{a^{\prime}(u)}, \\
C(x, t)= & -\rho(x)\left\{f^{\prime}(u) \Phi(u)-f(u) \Phi^{\prime}(u)-2 \rho^{\prime}(x) \Phi(u) \Phi^{\prime}(u)\right. \\
& \left.+\frac{2 \rho^{\prime}(x) a^{\prime \prime}(u) \Phi(u)^{2}}{a^{\prime}(u)}+\frac{\rho^{\prime \prime}(x) \Phi(u)}{\rho^{\prime}(x)}\right\}, \\
D(x, t)= & -\rho(x) \Phi^{\prime \prime}(u) u_{x}^{2}+\frac{\rho(x) a^{\prime \prime}(u) \Phi(u)}{a^{\prime}(u)}\left\{\rho(x)^{2} \Phi(u) \Phi^{\prime}(u)+f(u)\right\} .
\end{aligned}
$$

We additionally assume the following

(A9) For $a(\xi)$ and $f(\xi)$ there exists a function $\Phi(\xi)$ such that

$$
\begin{gathered}
\Phi(\xi), \Phi^{\prime}(\xi)>0 \text { and } \Phi^{\prime \prime}(\xi) \geq 0, \\
\int_{\xi}^{\infty} \frac{d \eta}{\Phi(\eta)}<\infty, \\
f(\xi) \Phi^{\prime}(\xi)-f^{\prime}(\xi) \Phi(\xi) \geq c\left\{\Phi(\xi) \Phi^{\prime}(\xi)-\frac{a^{\prime \prime}(\xi) \Phi(\xi)^{2}}{a^{\prime}(\xi)}\right\}
\end{gathered}
$$

for $\xi \geq \xi_{1}$, where $c=c\left(\xi_{1}\right)>0$. Moreover, for any $M>0$ there exists a $\xi_{2}=$ $\xi_{2}(M) \geq \xi_{1}$ such that

$$
f^{\prime}(\xi) \Phi(\xi)-f(\xi) \Phi^{\prime}(\xi) \geq M \Phi(\xi) \text { for } \xi \geq \xi_{2} .
$$

Remark (cf.,[6]). Suppose that

$$
\begin{gathered}
a^{\prime \prime}(\xi) \leq 0 \text { and } f^{\prime \prime}(\xi) \geq 0, \\
(1-\delta) f(\xi) f^{\prime \prime}(\xi) \geq f^{\prime}(\xi)^{2} \text { and }\left\{a^{\prime}(\xi) f(\xi)\right\}^{\prime} \geq 0
\end{gathered}
$$

in $\xi \geq \xi_{1}$, where $0<\delta<1$ and $\gamma>0$. Then (A9) is satisfied by the function $\Phi(\xi)=f(\xi)^{\delta}$ if we choose $c\left(\xi_{1}\right)=\frac{1-\delta}{\gamma+\delta} f\left(\xi_{1}\right)^{1-\delta}$.

Theorem 2.4. We assume (A4), (A8) and (A9). Let $T>0$ be the blow-up time of $u(x, t)$. Suppose that $(2.7)$ is satisfied for some $0<\tau<T$ and $\omega_{0}=$ $\left(x_{0}, y_{0}\right) \subset(0, L)$. Then there are no blow-up points of $u(x, t)$ in $\omega_{0}$. 
Proof. We shall show this theorem in the case $(2.7)^{\prime}$. Contrary to the conclusion, assume that $z \in \omega_{0}$ is a blow-up point of $u(x, t)$. Then by Lemma 2.3 we have

$$
\lim _{t \uparrow T x \in \omega} \inf _{t \uparrow} u(x, t)=\infty \text { for any } \omega=(\bar{x}, \bar{y}) \subset \subset\left(x_{0}, z\right) .
$$

We put in (2.11)

$$
\rho(x) \equiv \rho_{\varepsilon}(x)=\varepsilon \sin \frac{\pi}{\ell}(x-\bar{x}), \ell=\bar{y}-\bar{x},
$$

where $\varepsilon>0$ is a parameter. We choose $M=2(\pi / \ell)^{2}$ in (A9) and $\varepsilon_{0}=\ell c\left(\xi_{1}\right) / 4 \pi$. By (2.19) there exists $\tau \leq \bar{\tau}<T$ such that

$$
u(x, t) \geq \xi_{2} \text { in } \omega \times(\bar{\tau}, T) .
$$

Thus, as remarked in the beginning of $\S 1, u(x, t)$ is classical in $\omega \times(\bar{\tau}, T)$. Since $\rho(x)>0,\left|\rho^{\prime}(x)\right| \leq \varepsilon \pi / \ell$ and $\frac{\rho^{\prime \prime}(x)}{\rho(x)}=-(\pi / \ell)^{2}$ in $\omega$, this and the above conditions (A8), (A9) imply

$$
C_{\varepsilon}(x, t) \leq 0 \text { and } D_{\varepsilon}(x, t) \leq 0 \text { in } \omega \times(\bar{\tau}, T)
$$

for any $0<\varepsilon \leq \varepsilon_{0}$. Thus, $J=J_{\varepsilon}$ with $0<\varepsilon \leq \varepsilon_{0}$ satisfies

$$
\left[a^{\prime}(u) J\right]_{t}-J_{x x} \leq A_{\varepsilon}(x, t) J_{t}+B_{\varepsilon}(x, t) J \quad \text { in } \omega \times(\bar{\tau}, T) .
$$

We fix $0<\varepsilon \leq \varepsilon_{0}$ to satisfy

$$
\max _{x \in \bar{\omega}} u_{x}(x, \bar{\tau})+\varepsilon \max _{x \in \bar{\omega}} F(u(x, \bar{\tau}))<0 .
$$

Then it follows that

$$
J(x, \bar{\tau})<0 \text { in } \bar{\omega}
$$

Moreover, by (2.7)'

$$
\left\{\begin{array}{l}
J(\bar{x}, t)=u_{x}(\bar{x}, t)<0 \\
J(\bar{y}, t)=u_{x}(\bar{y}, t)<0 \text { in }(\bar{\tau}, T) .
\end{array}\right.
$$

$(2.21) \sim(2.23)$ and a maximum principle for heat equations imply

$$
J(x, t)<0 \text { in } \bar{\omega} \times(\bar{\tau}, T),
$$

or equivalently,

$$
-\frac{u_{x}(x, t)}{\Phi(u(x, t))}>\varepsilon \sin \frac{\pi}{\ell}(x-\bar{x}) \quad \text { in } \bar{\omega} \times[\bar{\tau}, t) .
$$

Integrate the both sides over $(\bar{x}, \bar{y})$. Then noting $(2.14)$, we have 


$$
\begin{aligned}
\int_{u(\bar{y}, t)}^{\infty} \frac{d \xi}{\Phi(\xi)} & \geq \int_{u(\bar{y}, t)}^{u(\bar{x}, t)} \frac{d \xi}{\Phi(\xi)} \\
& \geq-\int_{\bar{x}}^{\bar{y}} \frac{u_{x}(x, t) d x}{\Phi(u(x, t))}>\varepsilon \int_{\bar{x}}^{\bar{y}} \sin \frac{\pi}{\ell}(x-\bar{x}) d x
\end{aligned}
$$

for any $t \in(\bar{\tau}, T)$. However, this contradicts to (2.19).

Corollary 2.5. We assume (A4),(A8) and (A9) and consider the Neumann problem in $\Omega=(0, L)$. If we assume further that

$$
u_{0}^{\prime}(x)<0 \text { in } \Omega, u_{0}(L)>0 \text { and } u_{0}^{\prime}(0)=u_{0}^{\prime}(L)=0,
$$

the corresponding solution $u(x, t)$ blows up at a single point $x=0$.

Proof. The function $v=u_{x}$ satisfies the initial-boundary value problem

$$
\begin{cases}{\left[a^{\prime}(u) v\right]_{t}=v_{x x}+f^{\prime}(u) v} & \text { in }(0, L) \times(0, T) \\ v(0, t)=v(L, t)=0 & \text { in }(0, T) \\ v(x, 0)=u_{0}^{\prime}(x)<0 & \text { in }(0, L) .\end{cases}
$$

Thus, by means of the above-cited maximum principle we have

$$
v(x, t)=u_{x}(x, t)<0 \quad \text { in }(0, L) \times(0, T),
$$

and the above theorem applies with $\omega=(0, L)$.

\section{References}

[1] Aronson D.G., Crandall M.G. and Peletier L.A., Stabilization of solutions of a degenerate nonlinear diffusion problem, Nonlinear Anal, 6 (1982), 1001-1022.

[2] Barbu V., Nonlinear Semigroups and Differential Equations in Banach Spaces, Noordhoff International Publishing, Leyden, The Netherlands, 1976.

[3] Chen X.-Y. and Matano H., Convergence, asymptotic periodicity and finite-point blow-up in one-dimensional semilinear heat equations, to appear in J.Differential Equations.

[4] Cortazar C. and Elgueta M., Large time behaviour of solutions of a nonlinear reactiondiffusion equation, Houston J. Math., 13 (1987), 487-497.

[5] Friedman A. and McLeod B., Blow-up of positive solutions of semi-linear heat equations, Indiana Univ. Math. J., 34 (1985), 425-447.

[6] Fujita H. and Chen Y.-G, On the set of blow-up points and Asymptotic behaviours of blow-up solutions to a semilinear parabolic equation, Preprint 87-13, Univ. Tokyo.

[7] Galaktionov V.A., Kurdyumov S.P., Mikhailov A.P. and Samrskii A.A., On unbounded solutions of the Cauchy problem for the parabolic equation $u_{t}=\nabla\left(u^{\alpha} \nabla u\right)+u^{\beta}$, Dokl. Akad. Nauk SSSR, 252 (1980), 1362-1364.

[8] Giga Y. and Kohn R.V., Asymptotically self-similar blow-up of semilinear heat equations, Comm. Pure Appl. Math., 38 (1985), 297-319.

[9] Itaya N., A note on the blowup-nonblowup problems in nonlinear parabolic equations, Proc. Japan Acad., 55 (1979), 241-244.

[10] On some subjects related to the blowing-up problem in nonlinear parabolic equations, Lecture Notes in Num. Appl. Anal., 2 (1980), 27-38, Kinokuniya. 
[11] Lacey A.A., Mathematical analysis of thermal runaway for spacially inhomogeneous reactions, SIAM J. Appl. Math., 43 (1983), 1350-1366.

[12] Ladyzenskaja O.A., Solonokov V.A. and Ural'ceva N.N., Linear and Quasilinear Equations of Parabolic Type, Transl. Math., Monographs, 23 AMS Providence, R.I. 1968.

[13] Oleinik O.A., Kalashnikov A.S. and Chzou Yui-Lin, The Cauchy problem and boundary problems for equations of the type of nonlinear filtration, Izv. Akad. Nauk SSSR Ser. Math., 22 (1958), 667-704.

[14] Suzuki R., On blow-up sets and asymptotic behavior of interfaces of one dimensional quasilinear parabolic equations, preprint 1989. 
